\title{
Common impairments and functional limitations of HIV sequelae that require physiotherapy rehabilitation in the medical wards at Queen Elizabeth Central Hospital, Malawi: A cross sectional study
}

\author{
Gift Treighcy Banda, Grace Mwale, Michael \\ Chimwala, Linda Malimusi, Enock Chisati
}

Date Received: 21-Dec-2017
Revision Received: 17-Apr-2019
Date Accepted: 25-Apr-2019
Correspondence: Gift Treighcy
Banda
(gbbanda@stud.medcol.mw)
https://dx.doi.org/10.4314/mmj.v31i3.2

University of Malawi, College of Medicine, Physiotherapy Department, Blantyre, Malawi

\section{Introduction}

\section{Abstract}

The progression of human immunodeficiency virus (HIV) to acquired immune deficiency syndrome (AIDS) and the use of antiretroviral therapy (ART) have resulted in a range of complications, which affect different body systems and result in functional limitations and disabling impairments.

Objective

To investigate HIV-related impairments and functional limitations that require physiotherapy rehabilitation in patients admitted to the medical wards at Queen Elizabeth Central Hospital in Blantyre, Malawi.

Methods

This was a descriptive quantitative cross-sectional study involving the participation of 32 female (59.3\%) and 22 male (40.7\%) adults living with HIV and admitted to the medical wards at Queen Elizabeth Central Hospital. We collected data using a questionnaire that consisted of the World Health Organization Disability Assessment Schedule 2 (WHODAS2.0) and some demographic questions. Results were analysed using the Statistical Package for the Social Sciences (SPSS).

Results

Participation in society was the most frequently reported functional complication; the least common limitation was getting along with people $(70.3 \%, \mathrm{n}=38$ participants) and interaction with others $(24 \% ; \mathrm{n}=13)$. Shortness of breath $(55 \%$; $\mathrm{n}=30)$, muscle weakness $(44.4 \% ; n=24)$ and joint and muscle pain $(37 \% ; n=20)$ were the most commonly reported impairments. Participation restriction in the society affected both males $(77.3 \%)$ and females $(70.7 \%)$. The least common limitation that required physiotherapy for both males and females was getting along with people (26.6\% and $25 \%$, respectively). On average, male and female participants had a disability severity score of $48.5 \pm 4.6$ and $42.2 \pm 22.8$, respectively.

Conclusion

Our data showed that there is a clear need for early intervention to halt or delay the progression of complications to avoid severe disability; this is not only good for the patient but also for the socioeconomic state of the nation. Timely and full functional assessment, as well as referral of people living with HIV/AIDS for rehabilitation, is an important step forwards.

Key Words

HIV, disability, impairments, physiotherapy, functional limitations, functional complication

\section{Introduction}

The first dose of anti-retroviral therapy (ART) in Malawi was administered in $2003^{1}$. The introduction of ART led to a dramatic change in human immunodeficiency virus (HIV) from a life-limiting illness to a chronic condition which is now viewed as 'an illness characterised by episodic disabilities ${ }^{2,3}$. HIV progresses to acquired immunodeficiency syndrome (AIDS) and together these two conditions present with physical, metabolic and functional limitations resulting from either weakened immunity from the virus or the metabolism and interaction of the drugs administered ${ }^{4}$. In 2017, Malawi was home to approximately 15.4 million people and $10.6 \%$ of the population was living with HIV or AIDS ${ }^{5}$. HIV mostly affects females in sub-Saharan Africa ${ }^{6}$ and the majority of the disease burden in Malawi is borne by females ${ }^{5,7}$. An estimated prevalence of $58 \%$ of the population are living

\section{with $\mathrm{HIV}^{7}$.}

HIV has been classified by the World Health Organization (WHO) into four different stages, ranging from 1 to 4 (the higher the number, the weaker the immunity); these stages present with different symptoms which can vary from person to person ${ }^{8,9}$. The availability of ART treatment has led to a great reduction in mortality and morbidity, a reduction in health service utilization and an improvement in the quality of life among persons living with HIV $^{10}$. However, the impact of living with HIV infection over many years, coupled with antiretroviral toxicities, has created a complex pattern of healthcare needs. Moreover, complications manifest in many ways and require multi-disciplinary teamwork to ensure that quality care is being given to each patient ${ }^{2}$.

Most previous studies of HIV used a system-based approach to present complications and mostly focused on the medical 
diagnosis $^{11-13}$. Other studies focused only on the effects of HIV on individual body systems ${ }^{14,15}$. For example, one previous study in Malawi showed that $40-70 \%$ of people living with HIV and AIDS present with neurological complications manifesting as hemiplegic-type symptoms ${ }^{14}$ and that stroke was strongly associated with $\mathrm{HIV}^{14}$. HIV presents with opportunistic diseases such as tuberculosis (TB) that can affect both the pulmonary (i.e., pulmonary TB) and musculoskeletal systems (i.e., spinal TB) ${ }^{11}$. Pulmonary TB is the most common cardiorespiratory effect of HIV worldwide ${ }^{15}$.

The resultant comorbidities and conditions associated with HIV can result in functional limitations such as poor concentration, chronic fatigue, reduced endurance, shortness of breath, muscle wasting, weakness, incontinence, altered sensation, altered tone, stiff joints, hemiparesis and paraparesis ${ }^{16}$. A previous study of health-related quality of life in people living with HIV/AIDS found that $30.9 \%$ of the study population had mobility problems, $14.8 \%$ had self-care problems, $31.7 \%$ had difficulties with activities of daily living, $69.1 \%$ had difficulties with pain discomfort and $33 \%$ had anxiety or depression ${ }^{17}$. Another study summarised the prevalence of impairments, activity limitations and participation restrictions according to the stage of HIV and showed that diarrhoea, reduced libido, general body weakness, headaches and chronic fatigue were among the most frequently experienced impairments ${ }^{16}$.

Vigorous and moderate activities, sexual activities and household chores were the most frequently reported limitations and these were more common in individuals whose cluster of differentiation 4 (CD4) count was less than 200 (implying severe immunosuppression $)^{16}$ and significantly less in those whose CD4 count exceeded $500^{16}$. A different study reported that the most common manifestation of HIV infection leading to disability is the weakness resulting from the presence of chronic illness ${ }^{18}$. The authors of this previous study highlighted that virtually all patients with AIDS complain of fatigue as a major factor that prevents them from continuing vocational activities, hobbies and selfcare $^{18}$. Using the World Health Organization Quality of Life HIV BREF, another study revealed that the proportion of respondents (who were all HIV-reactive) with a low quality of life was highest in the domain of social relationships; this affected $64.6 \%$ of the study population ${ }^{19}$.

Physiotherapy rehabilitation can successfully address many of these HIV-related impairments and functional limitations. In fact, there are educational e-modules to support evidence-informed rehabilitation, including a version for sub-Saharan Africa $^{20}$. In addition, research priorities for HIV rehabilitation have been clearly established on several topics: episodic health and disability; aging with HIV across the life course; concurrent health conditions; access to rehabilitation and models of rehabilitation service provision; effectiveness of rehabilitation interventions; and enhancing outcome measurements in HIV and rehabilitation ${ }^{21}$. Broadly defined, rehabilitation is any service or health provider that may address or prevent impairments, limit activities or restrict social participation experienced by an individual ${ }^{20,22}$. The perceived roles of rehabilitation providers in subSaharan Africa in the context of HIV have also been well documented. In Kenya and Zambia, physiotherapists and occupational therapists described the role of rehabilitation largely as missed opportunities for improving the lives of adults and children, calling for a paradigm shift to bring rehabilitation into the HIV care continuum ${ }^{23}$.

In Malawi, the field of physiotherapy is still growing; physiotherapists are not first-line practitioners in this country ${ }^{24}$. This means that patients do not get referred for physiotherapy even though such intervention has been proven to be beneficial ${ }^{25}$. Consequently, there is a need to raise awareness to both medical and health personnel and the public on the importance of physiotherapy in addressing HIV-related impairments and functional limitations.

The main objective of this study was to identify the common HIV-related impairments and functional limitations that would benefit from physiotherapy. Our specific objectives were to: (1) identify impairments that are the leading causes of functional limitations, (2) to determine the HIV stage at which functional limitations occur and (3) to identify the gender affected most commonly by functional limitations.

\section{Materials and methods}

We conducted this study at Queen Elizabeth Central Hospital $(\mathrm{QECH})$ in Blantyre, Malawi. This facility is the largest hospital in Malawi and receives the most complicated referrals from local clinics and regional district hospitals. At the time of this study, QECH had four full-time qualified physiotherapists and six rehabilitation technicians, two of whom work as occupational therapy technicians. QECH is also a teaching hospital for students from the University Of Malawi, College of Medicine. This includes senior physiotherapy students who rotate regularly in different clinical placements. When these students graduate from the College, some continue to practice at $\mathrm{QECH}$ as interns in order to gain registration by the Medical Council of Malawi. The Physiotherapy Department at QECH receives both inand out-patients.

\section{Ethical considerations}

The study took a period of 4 weeks from 24 October to 21 November 2016. We obtained written permission to carry out this study from the Head of the Department of Medicine at QECH. We also sought an ethical clearance letter from the College of Medicine Research Ethics Committee (COMREC), along with an approval stamp from the Director of QECH. Understanding that HIV is a sensitive topic, we approached nurses to let us know of the patients who knew their reactive HIV status before we sampled our population. We then approached the potential participants and gave them information and details about the research. We also spoke to them in case they had questions that they felt we did not answer were not answered by the information sheets. After they agreed to be part of the study, the participants were then required to sign an informed consent form. We gave them an option to withdraw if they felt the need to do so.

We kept the participants' privacy by not using any personal information in the study. The researchers also signed a form pledging to protect the privacy and confidentiality of the research and its participants. To maintain confidentiality, we did not share the data collected with any third party that was not a part of the research team.

\section{Study population and sampling methodology}

The study population consisted of all HIV-reactive patients in the medical wards of $\mathrm{QECH}$ who were aware of their status and receiving ARTs. We obtained a sample size of 77 patients, calculated using the total number of patients 
who were HIV reactive in the four medical wards $(\mathrm{n}=96)$. To arrive at 77 potential participants, we used a stratified random sampling technique. To start with, we went through all inpatient files kept in the nurse's office to come up with a list of all those who were tested to be HIV reactive in each ward. We then came up with another list of only those who knew of their HIV status. From this list, it was evident that the female to male ratio was $3: 2$. We assigned numbers to all patients and placed these numbers in two separate boxes, one for males and another for females. Using the ratio, we randomly drew the number of males and females from the boxes (giving a final number of 77). Of the 77 patients in the identified study population, 54 participated and completed the study, 10 refused to sign the consent forms, 4 withdrew from the study, 5 were discharged before approach and 4 passed away.

\section{Eligibility criteria}

We included all patients admitted in the medical wards and tested positive for HIV. Patients were required to be between 16 and 60 years of age for inclusion because a previous study performed in this country showed that a high number of people in this age group were living with HIV/AIDS5. We excluded patients that did not provide consent, those who were unconscious and those aged outside of the 16-60-year range.

\section{Data collection method}

Data were collected using a questionnaire which comprised the World Health Organization Disability Assessment Schedule 2.0 (WHODAS2.0) ${ }^{26}$. The WHODAS 2.0 is a validated, practical, generic assessment instrument that can measure health and disability at a population level or in clinical practice ${ }^{27}$. The WHODAS 2.0 captures the level of function in six domains of life: cognitive (understanding and communicating), mobility (moving and getting around), getting along with people (interacting with others), life activities (domestic responsibilities, leisure, work and school), participation (joining in community activities) and participating in society. We also recorded information relating to medical diagnosis and experience of physiotherapy. We interviewed participants to report on the reasons why they had problems in certain areas if any, to determine any specific impairment. We recorded the reasons given on a separate sheet. These interviews took about 10-20 minutes to complete and we used the WHO classification for HIV staging to classify patients because CD4 count and viral load were unknown.

\section{Data management and analysis}

We coded data to represent patient's responses and entered these coded data into a Microsoft Excel 2013 spreadsheet (using the numbers assigned to each participant prior to data collection). We used the Statistical Package for the Social Sciences (IBM SPSS statistic 2011, Chicago, USA) to analyse the data using the WHO syntax in three steps. First, we summated the recoded items within each domain. Secondly, summated all scores in the six domains, Finally, we converted the summary score into a metric value ranging from 0 to 100, in which 0 represented no disability and 100 represented full disability. We used descriptive statistics to depict the distribution of activity limitation, participation restriction and impairments relevant to physiotherapy. We used bar graphs to represent the distribution of common complications, the leading causes of complications and to highlight the most disadvantaged gender by living with HIV. We used a frequency table to show the distribution of complications during each stage of HIV and a summary table to present the severity of disability for each gender.

\section{Results}

We enrolled 54 in-patients into our study. Of these, 59.3\% were female and $40.7 \%$ were male $(z=1.36$, $\mathrm{p}$ value: $>0.05)$. Only $11 \%$ of the participants had ever been referred to physiotherapy; all of these patients reported that they had received less than five physiotherapy sessions. None of the participants were in WHO HIV stage 1; most individuals were in stage $3(68.5 \% \mathrm{n}=37)$. Within stage 3 , there were similar numbers of males and females (Table 1).

\section{Table 1. The numbers of people in different WHO stages of HIV recruited into this study}

\begin{tabular}{|llll|}
\hline Gender & & \\
\hline Stage & Male $(\mathrm{n}=22)$ & Female $(\mathrm{n}=32)$ & Total \\
\hline 1 & $0(0 \%)$ & $0(0 \%)$ & $0(0 \%)$ \\
\hline 2 & $0(0 \%)$ & $5(9.3 \%)$ & $5(9.3 \%)$ \\
\hline 3 & $18(33.3 \%)$ & $19(35.2 \%)$ & $37(68.5 \%)$ \\
\hline 4 & $4(7.4 \%)$ & $8(14.8 \%)$ & $12(22.2 \%)$ \\
\hline Total & $22(40.7 \%)$ & $32(59.3 \%)$ & $54(100 \%)$ \\
& & & \\
\hline
\end{tabular}

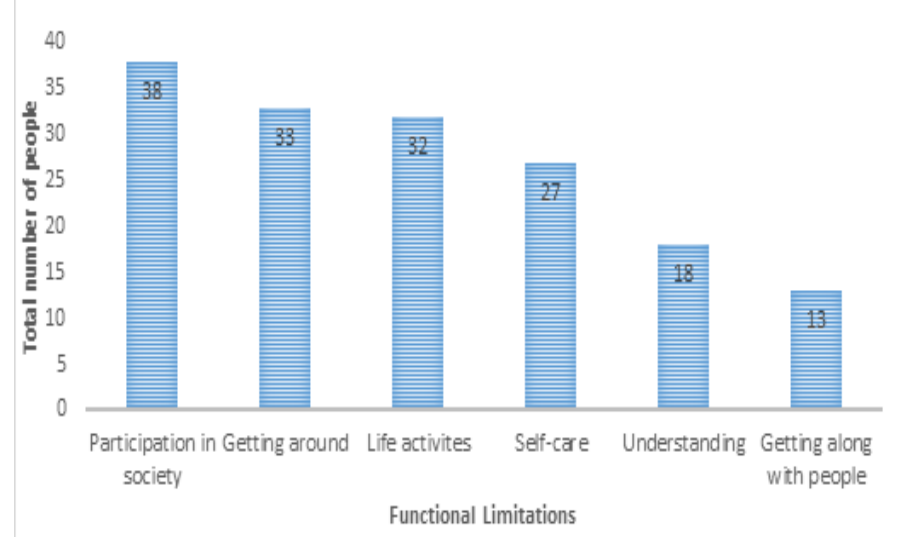

Figure 1: The total number of participants who had functional limitations

All 54 participants reported more than one complication; we classified and presented these according to the domains of the WHODAS2.0. The highest reported functional complication that required physiotherapy intervention was participation in society (38 participants, $70.6 \%$ ) while the least reported complication was getting along with people (13 people, 25.6\%) (Figure 1). We found that all 54 patients had experienced at least 2 impairments with regards to participation in society and restrictions in functional mobility Shortness of breath (55.5\%), muscle weakness (44.4\%) and joint and muscle pain (37\%) were the most commonly 


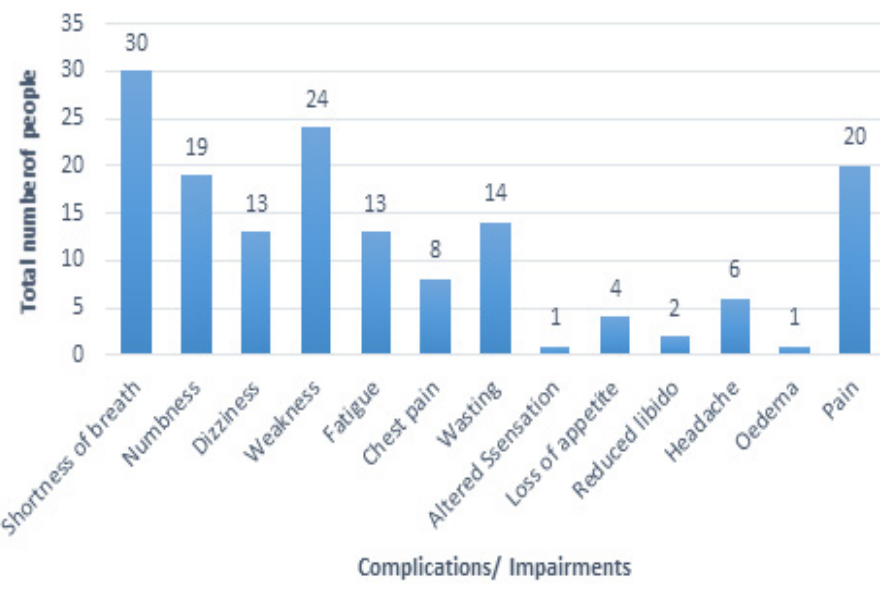

Figure 2: Leading impairments/complications in our study population which led to functional limitations

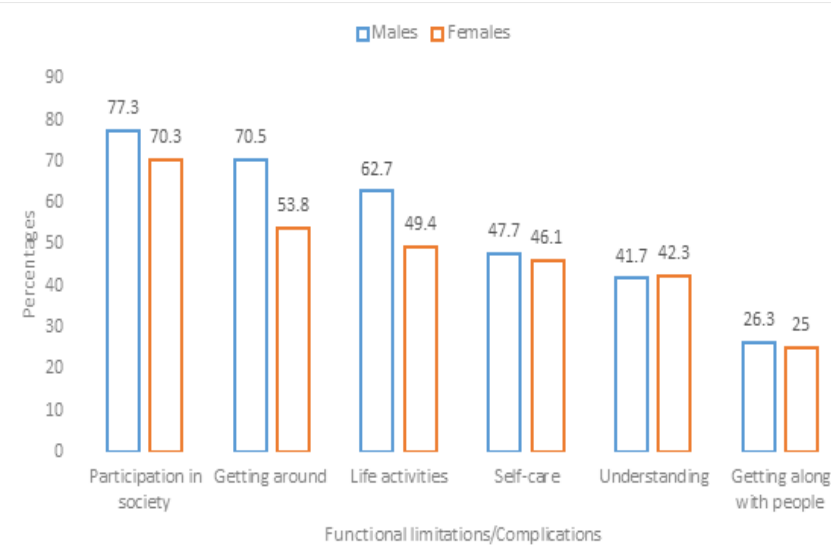

Figure 3: The proportions (\%) of participants with functional complications, separated by gender

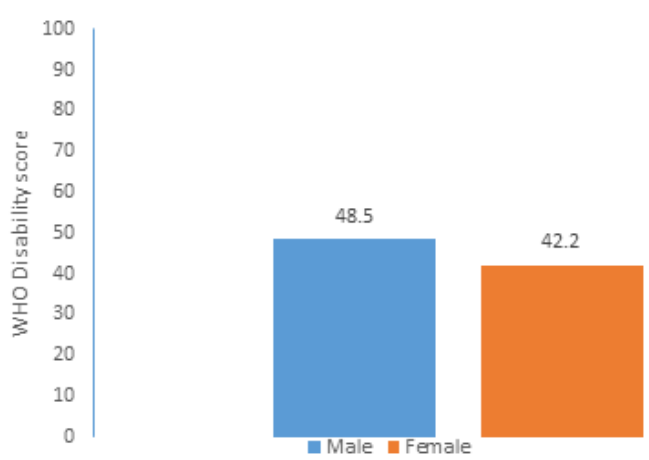

Figure 4: Mean disability scores for males and females

experienced impairments, while chest pain, oedema and altered sensation were the least reported (Figure 2).

Our results highlight that the issue of participation in society affected the male gender more than the female gender $(77.3 \%$ vs $70.7 \%$, respectively; $z=0.46, p$ value: 0.05$)$. The least reported limitation requiring physiotherapy intervention or rehabilitation for both males and females was getting along with people $(26.6 \%$ and $25 \%$, respectively). The frequencies of limitations related to understanding (cognitive) and selfcare (activities of daily living) were similar when compared between the two genders (Figure 3).

Using the WHODAS2.0 score, males scored 48.5 \pm 24.6 (mean \pm standard deviation) while females scored $42.2 \pm 22.6$ (Figure 4).

\section{Discussion}

This study shows that participation in society was the most common functional limitation among people living with
HIV/AIDS in Malawi; this was followed by getting around (functional mobility). Similar results were reported previously by Quinlivan et al. ${ }^{17}$ and Hughes et al. ${ }^{28}$. This was interesting because the patients reported by Hughes et al were not taking ARTs. Hanass-Hancock et al. ${ }^{29}$ also reported three studies in which activity limitations were the most predominant issue. We believe that in such cases, functional mobility is highly likely to affect the way such patients participate in society because for each patient, there is a requirement to be able to move and be available at certain places where participation is required. Such impairments can also have an impact on an individual's performance, which may result in functional limitation and physical disability ${ }^{30,31}$.

We also found that just over half of our participants had problems with self-care $(51.5 \% ; \mathrm{n}=27)$; these results were not consistent with a previous study carried out in Uganda, where self-care was the least reported problem ${ }^{32}$. One could attribute these differences to these studies focusing on different age groups, scope, demands and support systems. For example, the participants described in the previous study conducted in Uganda were not hospitalised, whereas our current patients were in-patients.

In a previous study, Rusch et al. identified that weakness and shortness of breath were the most prevalent impairments presented by people living with HIV / AIDS $^{16}$; these findings were similar to our present findings. Such impairments will affect the way in which patients will tolerate exercise and physiotherapy. We also found that muscle wasting was one of the most common impairments. In a previous study, HanassHancock et al. reported that $75 \%$ of their study population presented with muscle power loss ${ }^{9}$. One would expect this result because muscles are integral in carrying out activities of daily living ${ }^{23}$.

Our study also found that $24.1 \%$ of participants complained of fatigue. In the population of people living HIV/AIDS, it is known that reduced aerobic capacity is the leading cause of fatigue and physical disability ${ }^{30,31}$. Consequently, physiotherapists should consider this area carefully when planning a treatment program ${ }^{24,25}$.

The majority of our participants $(68.5 \% ; n=37)$ were classified as being in stage 3 of HIV; this was followed by stage $4(12.2 \% ; n=12)$. This is inconsistent with the study previously reported by Rusch et al. which noted that most complications and functional limitations were presented in patients in stage 416. However, our present results were similar to those reported by Hughes et al. ${ }^{28}$ in that most participants presenting with complications and functional limitations were in stages 3 and 4 . Interestingly, Rusch et al. conducted their study before ARTs became available. The introduction of ARTs delays disease progression, particularly when patients are compliant with their medication. However, stereotype and stigma towards HIV-positive patients remains very prevalent in society and thus prevents a proportion of the population taking an HIV test for fear of discrimination. We did not expect to recruit many patients in stage 1 because patients in this stage are not usually admitted into medical words as their symptoms are mostly asymptomatic and most people can carry out their activities of daily living without difficulty. However, exercise is still recommended for this group of people because it has been shown that there is a markedly reduced level of endurance among people living with $\mathrm{HIV} / \mathrm{AIDS}^{33}$.

In a previous study, Hanass-Hancock et al. concluded that it 
is crucial to understand the extent of disability among people living with HIV in high-prevalence settings in order to inform such patients of the best choices regarding care, policy and research $^{29}$. Since HIV mostly affects women in Malawi and all of sub-Saharan Africa ${ }^{5,6,34}$, we expected a larger number of females than males in our study. Our findings supported the results of a previous review by Hanass-Hancock et al. ${ }^{29}$ who found that almost all of the studies they reviewed recruited more females than males. Irrespective of this, there was a higher proportion of males in our study who reported complications with regards to participation restriction and activity limitations; similar results were reported by a Tanzanian study ${ }^{6}$. We anticipated that the proportion of complications would be the same for both genders, given that the progression of HIV is the same when compared between genders ${ }^{6}$. Interestingly, male participants presented with slightly higher disability severity in the cumulative domain scores (48.5/100); these results differed from those found in community dwelling participants in Uganda where females presented with a poorer health function status ${ }^{32}$.

\section{Limitations of the study}

The study has some limitations to consider. Firstly, this study features a small sample size. Consequently, our data may not be a true presentation of the complications faced on medical wards. We believe our small sample size was due to unwillingness of participants to enrol and because other participants dropped out of the study. Another limitation was that the WHODAS 2.0 measures perception and is therefore, a subjective tool, with consequential limitations. Furthermore, we must expect some levels of subjectivity concerning the interpretation of the meaning and ranking of some questions. This is because some words were difficult to understand, while some other words had no specific Chichewa translation. Finally, the precise evaluation of the severity of disability is not clearly indicated by the current WHO grading system, as interpretation of the rating of zero (no disability) to 100 (total disability) may be subjective.

\section{Recommendations}

We investigated the common HIV complications that require physiotherapy so that we could determine the stage of HIV at which these complications occur and the gender that these complications affects mostly likely. It was evident that participation in society and getting around were the most common functional complications that arise with HIV. We also found that shortness of breath and weakness were the leading causes of activity limitations and participation restriction. These complications appear in all stages of HIV but stage 3 presents with a significant proportion of complications. Many participants were also in stage 3 of the WHO grading system compared with the other stages. Furthermore, even though there were more males than females, complications affected the males more.

In Malawi, men are mostly the breadwinners in the home. Consequently, our results should be of huge concern because the higher rates of perceived disability in men could exert effect upon the socio-economic status of the country. Therefore, there is an urgent need for early intervention with which to halt or delay the progression of these complications to avoid severe disability.

We therefore recommend that health workers should assess every patient that has HIV for their functional status, regardless of the present medical diagnosis as patients all present with impairments across all stages of HIV, which can vary in extent. There is also a need for other medical team members to refer people living with HIV for physiotherapy.

\section{Authors' contributions}

GTB, GM, MC and LM worked together on this project in partial fulfilment of their Bachelor of Science in Physiotherapy (Honours) Degree at the University of Malawi, College of Medicine. EC critically revised the manuscript for important intellectual content. All authors agree and are accountable for all aspects of the work in ensuring that questions related to the accuracy or integrity of any part of the work are appropriately investigated and resolved.

\section{Acknowledgements}

We would like to thank our supervisor, Dr Vyvienne M'kumbuzi (the late Head of the Physiotherapy Department at the University of Malawi, College of Medicine) and $\mathrm{Mr}$ Reuben Kalavina. We also thank the Director, Head of Department of Medicine and the nurses at QECH for permitting us conduct this study at their facility and assisting us accordingly. We also thank Mr Andrew Kumitawa, Mr Vincent Samuel, Dr Hussein Twabi, Dr Parth Patel and Mr Fanuel Bickton for their insight on different areas of the study. Above all, thanks to the almighty God for being with us during this period.

\section{Funding}

The work described herein was jointly supported by the Africa Center of Excellence in Public Health and Herbal Medicine (ACEPHEM), College of Medicine, University of Malawi and Consortium for Advanced Research Training in Africa, (CARTA). ACEPHEM received a grant from the Malawi Government from an IDA Credit facility (ACE II) (P151847) Credit number 5802-MW. CARTA is jointly led by the African Population and Health Research Center and the University of Witwatersrand and funded by the Carnegie Corporation of New York (Grant No--B 8606.R02), Sida (Grant No: 54100029), the DELTAS Africa Initiative (Grant No: $107768 / Z / 15 / Z)$. The DELTAS Africa Initiative is an independent funding scheme of the African Academy of Sciences (AAS)'s Alliance for Accelerating Excellence in Science in Africa (AESA) and is supported by the New Partnership for Africa's Development Planning and Coordinating Agency (NEPAD Agency) with funding from the Wellcome Trust (UK) (Grant No: 107768/Z/15/Z) and the UK government.

\section{Availability of data and materials}

All data generated and analysed during this study are included in this published article. Its supplementary information files (including data collection tools and consent forms) are available from the corresponding author on reasonable request.

\section{Competing interests}

The authors have no conflict of interest.

\section{References}

1. Harries AD, Ford N, Jahn A, Schouten EJ, Libamba E, Chimbwandira F, et al. Act local, think global: how the Malawi experience of scaling up antiretroviral treatment has informed global policy. BMC Public Health. 2016;16:938. DOI: 10.1186/s12889-016-3620-x.

2. Chegwidden Wi, Rackstraw S, Duke K, Rich R, Noschese J, Mcdonnel E. Principles of physical and cognitive rehabilitation in HIV disease. In: Physical and Cognitive Rehabilitation Following Complications of https://dx.doi.org/10.4314/mmj.v31i3.2 
HIV Disease: Designing and Delivering Services in Different Settings Venue. Vienna: Internatinal AIDS Conference; 2010.

3. Brown D, Claffey A, Harding R. Evaluation of a physiotherapy-led group rehabilitation intervention for adults living with HIV referrals, adherence and outcomes. AIDS Care. 2016;28:1495-505. DOI: 10.1080/09540121.2016.1191611.

4. Simfukwe P. The role of physiotherapy in people living with HIV/ AIDS [internet]. 2019 [cited 2019 Feb 02]. Available from: http://www. kznhealth.gov.za/physio_hivaids.pdf.

5. ReliefTPEPFA. Malawi Country Operational Plan Strategic Direction Summary [Internet]. 2017 [cited 2019 Feb 02]. Available from: https:// www.pepfar.gov/documents/organization/272017.pdf.

6. Mosha F, Muchunguzi V, Matee M, Sangeda RZ, Vercauteren $\mathrm{J}$, Nsubuga $\mathrm{P}$, et al. Gender differences in HIV disease progression and treatment outcomes among HIV patients one year after starting antiretroviral treatment (ART) in Dar es Salaam, Tanzania. BMC Public Health. 2013;13:38. DOI: 10.1186/1471-2458-13-38.

7. AVERT. HIV and AIDS in Malawi [Internet]. Avert. 2014. p. 1-7 [cited 2019 Feb 02]. Available from: http://www.avert.org/aids-malawi. htm.

8. Center for Disease Control and Prevention. Stages of HIV Infection [Internet]. CDC. 2013. p. 1-3 [cited 2019 Feb 02]. Available from: https://www.aids.gov/hiv-aids-basics/just-diagnosed-with-hiv-aids/ hiv-in-your-body/stages-of-hiv/.

9. Interim WHO Clinical Staging of HIV/AIDS and HIV/AIDS case definitions for surveillance: African Region [Internet]. Geneva: World Health Organization; 2005. p. 20-8 [cited 2019 Feb 02]. Available from: http://www.who.int/hiv/en.

10. World Health Organization. Scaling up antiretroviral therapy in resource-limited settings: guidelinesfor a public health approach. 1st ed. Hammer S, Gibb D, Havlir D, Mofenson L, Van Beek I, Vella S, editors. Vol. 11, Infectious diseases in clinical practice. Geneva: World Health Organization; 2002. pp. 154-57. Available from: https://www. who.int/hiv/pub/prev_care/ScalingUp_E.pdf.

11. Myezwa H, Stewart A. HIV/AIDS in physiotherapy education mainstreaming using UN principles. S Afr J Physiother. 2012; 68(1): a7. DOI: $10.4102 /$ sajp.v68i1.7.

12. Boccara F. Cardiovascular complications and atherosclerotic manifestations in the HIV-infected population: type, incidence and associated risk factors. AIDS. 2008; Suppl 3:S19-26. DOI: 10.1097/01. aids.0000327512.76126.6e13.

13.Pozniak A, Rackstraw S, Deayton J, Barber T, Taylor S, Manji H, et al. HIV-associated neurocognitive disease: Case studies and suggestions for diagnosis and management in different patient subgroups. Antivir Ther. 2014;19:1-13. DOI: 10.3851/IMP2563.

14. Jung A, Raman A, Mallewa M, Solomon T. Neurology research and teaching in Malawi. Clin Med (Lond). 2009;9:570-1.

15. Kwan CK, Ernst JD. HIV and tuberculosis : a deadly human syndemic. Clin Microbiol Rev. 2011;24:351-376. DOI: 10.1128/ CMR.00042-10.

16. Rusch M, Nixon S, Schilder A, Braitstein P, Chan K, Hogg RS Impairments, activity limitations and participation restrictions: prevalence and associations among persons living with HIV / AIDS in British Columbia. Heal Qual Life Outcome. 2004;2:46. DOI: 10.1186/1477-7525-2-46.

17. Quinlivan EB, Fletcher J, Eastwood EA, Blank AE, Verdecias N, Roytburd K. Health status of HIV-infected women entering care: baseline medical findings from the Women of Color Initiative. AIDS Patient Care STDS. 2015;29(S1):S11-9. DOI: 10.1089/apc.2014.0277. review. Arch Phys Med Rehabil. 1991;72:690-2.

19. Imam M, Karim M, Ferdous C, Akhter S. Health related quality of life among the people living with HIV. Bangladesh Med Res Counc Bull. 2011;37:1-6. DOI: 10.3329/bmrcb.v37i1.7791.

20. Worthington C, Myers T, O'Brien KK, Nixon SA, Cockerill R. How rehabilitation can help people living with HIV in sub-Saharan Africa. E-Module SSA [Internet]. Pubmed. 2015 [cited 2019 Jan 19]. p. 258 71. Available from: http://ssa.hivandrehab.ca/.

21. O'Brien KK, Ibanez-Carrasco F, Solomon P, Harding R, Cattaneo $\mathrm{J}$, Chegwidden W, et al. Advancing research and practice in HIV and rehabilitation: a framework of research priorities in HIV, disability and rehabilitation. BMC Infect Dis. 2014;14:724. DOI: 10.1186/s12879014-0724-8.

22. Nixon SA, Forman L, Hanass-Hancock J, Mac-Seing M, Munyanukato N, Myezwa H, et al. Rehabilitation : a crucial component in the future of HIV care and support. South Afr J HIV Med. 2011;12(2):12-17.

23. Nixon S, Cameron C, Mweshi M, Nkandu EM, Okidi C, Tattle S, et al. "It Is an eye-opener that there is a relationship between rehabilitation and HIV": Perspectives of physiotherapists and occupational therapists in Kenya and Zambia on the role of rehabilitation with adults and children living with HIV. Physiother Can. 2016;68:290-8. DOI: $10.3138 /$ ptc. $2015-42 \mathrm{GH}$.

24. Mpezeni S, Benjamin L, Cary I. Point of view : Physiotherapy in Malawi - a step in the right direction. Malawi Med J. 2013;25:83-5.

25. Pullen SD, Chigbo NN, Nwigwe EC, Chukwuka CJ, Amah CC, Idu SC. Physiotherapy intervention as a complementary treatment for people living with HIV/AIDS. HHIV AIDS (Auckl). 2014; 6: 99-107. DOI: $10.2147 /$ HIV.S62121.

26. Üstün TB, Kostanjsek N, Chatterji S, Rehm J. Measuring health and disability manual for WHO disability assessment schedule. 1st ed. Unstun T, Kostanjsek N, Chatterji S, Rehm J, editors. Geneva: World Health Organization; 2010. pp. 47-58.

27. Moen VP, Drageset J, Egil G, Klokkerud M. Validation of World Health Organization assessment schedule 2.0 in specialized somatic rehabilitation services in Norway. Qual Life Res. 2016;26:505-14. DOI: $10.1007 / \mathrm{s} 11136-016-1384-5$.

28. Hughes J, Jelsma J, Darder M, Tinise X. The health-related quality of life of people living with HIV/AIDS. Disabil Rehabil. 2004;26:3716. DOI: $10.1080 / 09638280410001662932$

29. Hanass-Hancock J, Regondi I, van Egeraat L, Nixon S. HIV-related disability in HIV hyper-endemic countries : a scoping review. World J AIDS. 2013;3:257-279. DOI: 10.4236/wja.2013.33034 30.

30. Cade WT, Peralta L, Keyser RE. Aerobic exercise dysfunction in human immunodeficiency virus: a potential link to physical disability. Phys Ther. 2004;84:655-64. https://DOI.org/10.1093/ptj/84.7.655.

31. Useh U. HIV and AIDS : its implication for physiotherapy practice and exercise prescription. J Hum Ecol. 2013;42):135-140.

32. Scholten F, Seeley J, Kinyanda E, Nakubukwa S, Kowal P, Naidoo N, et al. Health and functional status among older people with HIV/AIDS in Uganda. BMC Public Health. 2011;11:886. DOI: 10.1186/14712458-11-886.

33. Chisati EM, Vasseljen O. Aerobic endurance in HIV-positive young adults and HIV-negative controls in Malawi. Malawi Med J. 2015;27:5-9.

34. Griesbeck M, Scully E, Altfeld M. Sex and gender differences in HIV-1 infection. Clin Sci (Lond). 2016;130:1435-51. DOI: 10.1042/ CS20160112. 